\title{
Comparative transcriptome analysis revealed genes involved in the fruiting body development of Ophiocordyceps sinensis
}

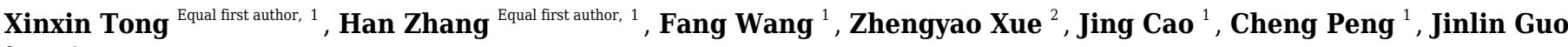 \\ Corresp. 1 \\ ${ }^{1}$ Key Laboratory of Standardization of Chinese Medicine, Ministry of Education; Key Laboratory of Systematic Research, Development and Utilization of \\ Chinese Medicine Resources in Sichuan Province-Key Laboratory Breeding Base founded by Sichuan Province, Chengdu University of Traditional Chinese \\ Medicine, Chengdu, Sichuan, China \\ 2 Department of Food Science and Technology, University of California, Davis, Davis, CA, United States \\ Corresponding Author: Jinlin Guo \\ Email address: guo596@cdutcm.edu.cn
}

Ophiocordyceps sinensis is a highly valued fungus that has been used as traditional Asia medicine. This fungus is one of the most important sources of income for the nomadic populations of the Tibetan Plateau. With global warming and excessive collection, the wild $O$. sinensis resources declined dramatically. The cultivation of $O$. sinensis hasn't been fully operational due to the unclear genetic basis of the fruiting body development. Here, our study conducted pairwise comparisons between transcriptomes acquired from different growth stages of $O$. sinensis including asexual mycelium (CM), developing fruiting body (DF) and mature fruiting body (FB). All RNA-Seq reads were aligned to the genome of $O$. sinensis CO18 prior to comparative analyses. Cluster analysis showed that the expression profiles of FB and DF were highly similar compared to $\mathrm{CM}$. Alternative splicing analysis (AS) revealed that the stage-specific splicing genes may have important functions in the development of fruiting body. Functional enrichment analyses showed that differentially expressed genes (DEGs) were enriched in protein synthesis and baseline metabolism during fruiting body development, indicating that more protein and energy might be required for fruiting body development. Besides, some fruiting body developmentassociated genes impacted by ecological factors were up-regulated in FB samples, such as nucleoside diphosphate kinase gene ( $n d k), \beta$ subunit of fatty acid synthase gene (cel-2) and superoxide dismutase gene (sod). Moreover, the expression levels of several cytoskeletons genes were significantly altered during all these growth stages, suggesting that these genes play crucial roles in both vegetative growth and the fruiting body development. Quantitative PCR (qPCR) was used to validate the gene expression profile and the results supported the accuracy of the RNA-Seq and DEGs analysis. Our study offers a novel perspective to understand the underlying growth stage-specific molecular differences and the biology of $O$. sinensis fruiting body development. 


\title{
1 Comparative Transcriptome Analysis Revealed Genes Involved in the Fruiting Body \\ 2 Development of Ophiocordyceps sinensis
}

\author{
3 Xinxin Tong ${ }^{1 \wedge}$, Han Zhang ${ }^{1 \wedge}$, Fang Wang ${ }^{1}$, Zhengyao Xue ${ }^{2}$, Jing Cao ${ }^{1}$, Cheng Peng ${ }^{1}$ and Jinlin Guo ${ }^{1 *}$ \\ 4 1. Key Laboratory of Standardization of Chinese Medicine, Ministry of Education; Key Laboratory of \\ 5 Systematic Research, Development and Utilization of Chinese Medicine Resources in Sichuan Province - \\ 6 Key Laboratory Breeding Base founded by Sichuan Province; Chengdu University of Traditional \\ 7 Chinese Medicine, Chengdu, 611137, China \\ 8 2. Department of Food Science and Technology, University of California, Davis, CA, 95616,USA \\ $9 \quad{ }^{*}$ Corresponding Author: guo596@cdutcm.edu.cn \\ $10 \wedge$ These two authors contribute equally to this work.
}

\begin{abstract}
Ophiocordyceps sinensis is a highly valued fungus that has been used as a traditional Asia medicine. This fungus is one of the most important sources of income for the nomadic populations of the Tibetan Plateau. With global warming and excessive collection, the wild O. sinensis resources declined dramatically. The cultivation of $O$. sinensis hasn't been fully operational due to the unclear genetic basis of the fruiting body development. Here, our study conducted pairwise comparisons between transcriptomes acquired from different growth stages of O. sinensis including asexual mycelium (CM), developing fruiting body (DF) and mature fruiting body (FB). All RNA-Seq reads were aligned to the genome of O. sinensis CO18 prior to comparative analyses. Hierarchical clustering analyses showed that the expression profiles of FB and DF were highly similar compared to CM. Alternative splicing analysis (AS) revealed that differential splicing genes (DSGs) might have important functions in fruiting body development. Functional enrichment analyses showed that differentially expressed genes (DEGs) were enriched in protein synthesis and baseline metabolism during fruiting body development, indicating that more protein and energy might be required for fruiting body development. Besides, some fruiting body development-associated genes impacted by ecological factors were up-regulated in FB samples, such as nucleoside diphosphate kinase gene $(n d k), \beta$ subunit of fatty acid synthase gene (cel-2) and superoxide dismutase gene (sod). Moreover, the expression levels of several cytoskeletons genes were significantly altered during these growth stages, suggesting that these genes play crucial roles in both vegetative growth and the fruiting body development. Quantitative PCR (qPCR) was used to validate the gene expression profile and the results supported the accuracy of the RNA-Seq and DEGs analysis. Our study offers a novel perspective to understand the underlying growth stage-specific molecular differences and the biology of $O$. sinensis fruiting body development.
\end{abstract}

Keywords: Ophiocordyceps sinensis; comparative transcriptome analysis; fruiting body development

\section{Introduction}

Peer) reviewing PDF | (2019:08:40454:3:0:NEW 26 Nov 2019) 
Ophiocordyceps sinensis (Berk.), belonging to Ascomycetes, is endemic to the Tibetan Plateau and the Himalayas with high latitudes from 3000 - $5000 \mathrm{~m}$ (Lo 2015). The Chinese name for O. sinensis is “Dong Chong Xia Cao", meaning "winter worm, summer plant". The name derived from its unusual lifestyle: entering and growing inside the ghost moth caterpillars (Thitarodes spp.), developing the fruiting body from the caterpillar shell, and forming a parasitic fungus-insect complex that comprises a mature fruiting body with perithecium (Lo 2015). A full life cycle of O. sinensis is typically 3-5 years when growing in the wild (Qilian Qin 2018). At present, the molecular basis of fruiting body development hasn't been fully understood.

O. sinensis has been widely used as a traditional Asian medicine to treat asthma, bronchitis, lung inflammation, nocturnal emissions, night sweats, and other diseases for over 2000 years (Jin Xu 2016). This medicinal fungus is also one of the most important sources of income for the native tribes of the Tibetan Plateau (Pöggeler 2006; Jinlin Guo 2012; Enhua Xia 2017). Due to the high medical value and huge market demand, the price of mature fruiting body was as high as approximately USD 60,000 per $\mathrm{kg}$ for the top grade in 2015 (Qilian Qin 2018). The natural populations of O. sinensis were overexploited, inevitably impairing the sustainability of the regional economy of Tibet and endangering this species (Qilian Qin 2018). Even though under the optimized artificial cultivation, the fruiting body takes 1-2 years to mature (Qilian Qin 2018). To date, the large-scale cultivation of $O$. sinensis has not been realized. A better understanding the biology of the fruiting body development is essential for scaling up the cultivation of $O$. sinensis to meet the medicinal demand and reduce the exploitation of wild $O$. sinensis populations.

Fruiting body development of $O$. sinensis requires special environmental factors and has been demonstrated to be initiated by the ecological factors specific to the alpine ecosystem, such as high latitude, low temperature and intensive solar ultraviolet (UV) radiation (Enhua Xia 2017). The influence of environmental factors on fruiting body development has been investigated in some Ascomycetes fungi, including Neurospora crassa and Pestalotiopsis microspore, etc. (Oda K 1997; Lee JI 2004; Pöggeler 2006). Previous studies showed that both orientation and the position of the neck on the perithecium were lightdependent in N. crassa (Oda K 1997). Light controls the balance of asexual versus sexual reproduction of Aspergillus nidulans (Busch S 2010). Moreover, several genes involved in the perception of these environmental factors have been identified, such as nucleoside diphosphate kinase gene and a subunit of COP9 signalosome gene (Oda K 1997; Busch S 2010). These results provide good references for researches in O. sinensis.

With the development of high throughput sequencing technology, the genomes and transcriptomes of O. sinensis and related species have been analyzed (Xin Zhong 2016; Xin Zhong 2018). Recent research detected the signals of the positive selection for genes involved in peroxidase and hypoxia to enable the highland adaptation by sequencing 31 whole genomes of $O$. sinensis from different distributions (Enhua Xia 2017). Li Xiang et al. constructed an O. sinensis EST database by using Roche/454 GS-FLX (Titanium) pyrosequencing technology, 4 mating type genes and 121 genes putatively associated with fruiting body development were identified (Li Xiang 2014). Moreover, the transcripts of three developmental stages (mycelia, sclerotium and fruiting body) were sequenced and compared, however, both sclerotium and fruiting body were the worm - part and grass - part of O. sinensis respectively, which couldn't represent the different growth stages of $O$. sinensis (Xin Zhong 2018). Transcriptome analysis of O. sinensis before and 
after infection of Thitarodes offered insights into the infection biology by which the fungus enters into the host partner (Xin Zhong 2016).

Built on prior results, in this study, the samples of $O$. sinensis were all harvested from artificial cultivation in our lab, which enabled a convenient sampling of asexual mycelium, the developing stroma and mature fruiting body. With the state-of-art Illumina HiSeq 2000 technology, transcriptome profiles of the three growth stages were sequenced in two biological replicates and compared between different growth stages. Our study revealed significant differences in expression profiles of genes involved in protein biosynthesis and baseline metabolism in these growth stages. Moreover, some fruiting body development associated genes influenced by environmental factors were identified. For the first time, the expression levels of cell cytoskeleton genes were altered throughout the growth stages in this study, indicating that cytoskeletons might be critical for fruiting body formation. Besides, stage-specific splicing genes might play an important roles in fruiting body development. Together, our study provided novel insights into the genetic basis of fruiting body development, which would facilitates the large-scale artificial cultivation of O. sinensis.

\section{Materials and Methods}

\subsection{Specimen collection, RNA extraction and Sequencing}

Three developmental stages of $O$. sinensis were artificially cultivated and collected in our lab. O. sinensis strain was deposited in the China General Microbiological Culture Collection center, accession No. CGMCC 3.14243, and cultured on potato dextrose agar (PDA) plate. The asexual mycelium (CM) from PDA plate was harvested for RNA isolation. The samples of stroma without asci was designated as the developing fruiting body (DF). The samples of fruiting body with asci and ascospores were designated as mature fruiting body (FB) and harvested for RNA isolation. All fresh specimen collected were stored in RNAlater ${ }^{\circledR}$ (Ambion, Austin, Texas, USA) according to the manufacturer's instruction until ready for RNA extraction. Total RNA was isolated by using TRIzol (Invitrogen, USA) according to the manufacturer's instruction. Genomic DNA was digested by DNase I (Fermentas, USA). Purified RNA was electrophoresed in a 1\% agarose gel, and the purity and the quality of RNA were assessed by OD260 and OD230. At least $20 \mu \mathrm{g}$ total RNA was then submitted to Biomarker Technology Co., Ltd (Beijing, China) for quality control using an Agilent Bioanalyzer 2100 (Agilent Technologies, CA, USA).

RNA purity, concentration and RNA integrity number (RIN) were measured by Agilent 2100 Bioanalyzeror SMA3000. The mRNA was enriched from total RNA using poly (T)+ oligo attached magnetic beads, eluted with Tris-HCl buffer and fragmented in fragmentation buffer using an RNA fragmentation kit (Ambion, Austin, TX, USA). First-strand cDNAs were reverse transcribed with reverse transcriptase and random hexamer-primers, and then second-strand cDNAs were synthesized by DNA Polymerase I.A single ' $A$ ' base was added to the ends of these dscDNA, and then the cDNA was ligated with Illumina sequencing adaptors and separated in an agarose gel. The $200 \mathrm{bp}$ cDNA fragments were then purified from the gel using Gel Extraction Kit (Axygen, Biosciences, Central Avenue Union City, CA, USA) for further library preparation using NEB Next Ultra RNA Library Prep Kit for Illumina (NEB, USA) according to the manufacture's instruction. The fragmented cDNA libraries of each sample were then submitted to an 
113

114

115

116

117

118

119

120

121

122

123

124

125

126

127

128

129

130

131

132

133

134

135

136

137

138

139

140

141

142

143

144

145

146

147

148

149

150

Illumina HiSeq 2000 platform at Biomarker Technology Co., Ltd. (Beijing, China) for transcriptome sequencing with the $125 \mathrm{bp}$ paired-end (PE) chemistry. Adapter sequence and low quality bases were removed from the raw reads.

\subsection{Read mapping, annotation and differential expression analysis}

O. sinensis genome was downloaded from the NCBI database (https://www.ncbi.nlm.nih.gov/assembly/GCA_000448365.1/). Trimmed paired-end reads of FB, DF and $\mathrm{CM}$ were aligned to the reference genome by the fast, splice-aware HISAT2 aligner (version 2.0.4) (Kim 2015). Reads that have been aligned to the genome from each sample were then assembled into transcripts by StringTie (version 1.3.3b) using default parameters (version 2.0.6) (Ben Langmead 2010; MihaelaPertea 2015).The mapping results are shown in Table 1. Coding sequences were predicted by Transdecoder (version 2.0.1). Functional annotation of assembled sequences was retrieved from the databases of Kyoto Encyclopedia of Genes and Genomes (KEGG, http://www. kegg.jp/ ) (Kanehisa M 2000), Cluster of Orthologous Groups (COG, http://www.ncbi.nlm.nih.gov/COG/ ) (Ashburner M 2000; Tatusov RL 2003), NCBI's non-redundant protein (NR, http://www.ncbi.nlm.nih.gov/sites/entrez?db=protein ) and SwissProt ( http://www. expasy.org ). To globally characterize the expression patterns of diverse RNA-Seq samples, paired-end reads were aligned back to the assembled transcripts using Bowtie 2.0 as the aligner (Langmead B 2012). Gene expression patterns were quantified using STAR-RSEM algorithm (version 4.1) based on the read numbers that were mapped to each gene. The mapped read numbers of each assembled transcript were calculated, and were normalized by RESM-based algorithm to get FPKM values for each RNA-Seq sample using perl scripts in the Trinity package (MihaelaPertea 2016). RSEM results of each replicate of the sample were merged as one matrix for downstream analyses. The new assembled transcripts were deposited into FigShare ( https://figshare.com/s/aca5204d75581bb3dec8).

Using the edgeR package (Empirical analysis of Digita Gene Expression in R, http://www.bioconductor.org/packages/elease/bioc/tml/edgeR. html), differentially expressed genes (DEGs) were identified with a threshold of $\mid \log _{2} \mathrm{FC}$ ( $\log _{2}$ fold-change) $\mid \geq 2$ and false discovered rate $(\mathrm{FDR})<0.001$ as statistically significant. With the TopGO package (http://www.bioconductor.org/packages/release/bioc/html/topGO.html), enrichment was tested using a hypergeometric test with a threshold value of $\mathrm{p} \leq 0.01$ for GO functions and KEGG pathways. The expression patterns of DEGs across the growth stages were further clustered using hierarchical clustering implemented in R package. For each cluster, GO term enrichment analysis was conducted using TopGO package with a threshold value of $\mathrm{p} \leq 0.01$.

Raw Illumina sequencing results of $O$. sinensis were submitted to NCBI Sequence Read Archive (SRA) with the accession numbers: SRR5282569, SRR5282570, SRR5282574, SRR5282575, SRR5282577and SRR5282578.

\subsection{Alternative Splicing Prediction}

ASprofile (version b-1.0.4, http://ccb.jhu.edu/software/ASprofile/ was used to detect the splice junction sites and provided information about boundaries and combinations of different exons in a transcript, and 
151

152

153

154

155

156

157

158

159

160

161

162

163

164

165

166

167

168

169

170

171

172

173

174

175

176

177

178

179

180

181

182

183

184

185

then total splice junction sites of the same gene were used to distinguish the type of its alternative splicing (AS) event. The ASprofile analysis was deposited into FigShare (DOI: 10.6084/m9.figshare.11086478). Using rMATs (version 4.0.3, http://rnaseq-mats.sourceforge.net/), the number of differential splicing events (DASs) and different splicing genes (DSGs) were compared between different growth stages and FDR < 0.05 as statistically significant. TopGO was used to perform function enrichment analysis of DSGs between different growth stages and $\mathrm{p} \leq 0.01$ was used to judge the significant enriched GO terms.

\subsection{Quantitative RT-PCR}

Ten genes that were randomly selected between the three growth stages according to the transcriptome sequencing analysis were selected and validated by qPCR experiments in triplicate. Primers were designed according to the sequences acquired from Illumina sequencing results with Primer Premier 5.0. The specific primer sequences are listed in Supplemental information 11. Aliquots of cDNA template submitted for Illumina HiSeq sequencing were used for qPCR analysis.. cDNA amplification was performed in a total of $25 \mu \mathrm{L}$ reaction system containing $12.5 \mu \mathrm{L} 2 \times$ UltraSYBR qPCR Mix (Cwbiotech, Co., LTD, Beijing, China), $2 \mu \mathrm{L}$ of diluted cDNA mix, $0.5 \mu \mathrm{L}$ of each primer $(10 \mathrm{mM})$. The cycling conditions were as follows: $95{ }^{\circ} \mathrm{C}$ for $10 \mathrm{~min}, 40$ cycles of $95{ }^{\circ} \mathrm{C}$ for $10 \mathrm{~s}, 57^{\circ} \mathrm{C}$ for $15 \mathrm{~s}$ and $72{ }^{\circ} \mathrm{C}$ for $20 \mathrm{~s}$. Relative gene expression levels were analyzed by the $2^{-\Delta \Delta \mathrm{Ct}}$ method. The histone $2 \mathrm{~A}$ gene (MSTRG. 2474) was used to normalize the expression level. Graphpad Prism program (version 5.0, GraphPad Software, Inc.) was used for statistical analysis.

\section{Results}

\subsection{RNA sequencing and mapping}

To investigate the profile of gene expression during growth, samples with two biological replicates from the stages of (CM, DF and FB) were submitted for RNA-Seq. After cleaning and quality control, more than 63 million paired-end, clean reads were obtained for each replicate with Illumina HiSeq. Reads of CM, DF and FB were aligned to the O. sinensis CO18 genome using HISAT2(Kim 2015), and assembled by StringTie (MihaelaPertea 2015), which resulted in 11,497 genes, including 7518 protein coding genes and 233 putative novel genes (Supplementary information 1). The number of aligned reads per gene was normalized by the STAR-RSEM based algorithm (Li B 2011). For each replicate, over $80.77 \%$ reads were successfully mapped (Table 1). In a previous study, Li Xiang et al. constructed an O. sinensis EST database using Roche / 454 GS-FLX (Titanium) pyrosequencing technology and they obtained a total of 1,743,676 high-quality reads with an average length of $426 \mathrm{bp}$, and assembled these reads into 34,289 unique sequences with an average length of $612 \mathrm{bp}$ (Li Xiang 2014). BLAT comparison was performed between the 11,497 genes in this study and 34,289 sequences in Li's study (Li Xiang 2014), which revealed that more than $90.00 \%$ of the 34,289 sequences can be found in the 11,497 genes with an identity of $90 \%$, while only $78.01 \%$ of the 11,497 genes can be found in the 34,289 sequences, indicating that our assembly identified more genes than the previous study.

\subsection{Differential gene expression and functional enrichment analysis}

Peer) reviewing PDF | (2019:08:40454:3:0:NEW 26 Nov 2019) 
DEGs were considered statistically significant if the $\mid \log _{2} \mathrm{FC}$ ( $\log _{2}$ fold-change) $\mid$ is more than 2 and the FDR is less than 0.001. This threshold resulted in a total of 1153 genes as significant DEGs in DF vs. CM , 503 in FB vs. DF and 1234 in FB vs. CM (Fig. 1A, Supplemental information 1). There were 19 shared DEGs among the three comparisons of the different growth stages (Fig.1B, Supplemental information 2). Among them, one gene (MSTRG.3745) encoding one hypothetical protein with the homology of 60s ribosomal protein L3 in O. sinensis CO18 was significantly up-regulated in FB compared to that in both $\mathrm{CM}$ and DF. However, most of the shared genes encode functionally unknown proteins, which might be related to fruiting body development and required further study to verify their functionalities.

Based on both KEGG and GO databases, we analyzed the enrichment of the DEGs across the three growth stages (Supplemental information 3). In DF vs. CM comparison, a total of 98 DEGs were mainly enriched in 'MCM complex', 'ATP catabolic process', 'ATP-dependent helicase activity', 'ATP binding', DNA replication initiation', 'replication compartment', and 'small-subunit processome' etc., among which 78 and 20 genes were up-regulated and down-regulated in DF, respectively (Supplemental information 3, 4), suggesting that the increased demand of energy and protein for fruiting body formation compared to the asexual mycelium stage. In FB vs. DF comparison, 161 DEGs were enriched in ' ribosome biogenesis', 'structural constituent of ribosome', 'ribonucleoprotein complex', 'MCM complex', 'translation', etc. were enriched (Fig. 2, Supplemental information 3), among which 103 and 58 genes upand down- regulated in FB, respectively (Supplemental information 4). Moreover, a total of 23 genes enriched in ribosome biogenesis were all significantly up-regulated in FB compared to DF (Supplemental information 3). Besides, in FB vs. CM comparison, 304 genes were mainly enriched in 'structure constituent of ribosome', 'ribosome biogenesis', 'translation', 'small ribosomal subunit', 'oxidoreductase activity', 'carbohydrate metabolic process', 'pyridine - containing /organic hydroxy compound biosynthetic process' and 'iron ion binding', etc. (Fig. 2, Supplemental information 3), in which 132 and 172 genes were up-regulated and down-regulated in FB, respectively ( Supplemental information 4).

\subsection{Clustering of gene expression profiles across three growth stages}

To verify the correlation between the expression of all the DEGs of developmental stages, principle component analysis (PCA) showed that FB shared more similar expression profiles with DF than CM (Fig. 3A). Furthermore, hierarchical clustering was performed on the basis of the expression level of DEGs across these growth stages, revealing that the three growth stages with two biological replicates could be assigned into two groups with FB and DF as one group and CM as the other (Supplemental information 5) and overall gene expression pattern was divided into seven gene clusters with visibly different profiles (Fig. 3B-H). Cluster 1 and cluster 3, with 712 and 72 transcripts, respectively, had a sudden decrease in expression upon transition from $\mathrm{CM}$ to $\mathrm{DF}$, and a steady trend from $\mathrm{DF}$ to $\mathrm{FB}$, indicating that these genes were up-regulated in CM and might play crucial roles in the growth of mycelium. Cluster 4, with 188 transcripts, had a steady trend from CM to DF, and then a sudden decrease in expression from DF to FB, illustrating that these genes mainly were expressed in CM and DF. Cluster 1, 3 and 4 were mainly enriched in genes involved in 'carbohydrate metabolic process', 'oxidoreductase activity' and 'protein kinase activity', which might contribute to the vegetative growth and physiological metabolism (Supplemental information 6). Cluster 2, with 139 transcripts, had a sudden decrease in expression upon shifting from CM 
225

226

227

228

229

230

231

232

233

234

235

236

237

238

239

240

241

242

243

244

245

246

247

248

249

250

251

252

253

254

255

256

257

258

259

260

261

262

to DF and a sudden increase from DF to FB. Cluster 6, with 280 transcripts, had a sudden increase in expression from $\mathrm{CM}$ to $\mathrm{DF}$, and a sudden decrease from $\mathrm{DF}$ to $\mathrm{FB}$, which indicated that these genes have different roles during the three stages. Cluster 5, with 471 transcripts, remained a stead trend from CM to $\mathrm{DF}$, and then a sudden increase in expression upon transition from DF to FB. Cluster 5 mainly consisted of genes involved in 'ribosomal structure constituent / biogenesis', 'translation', 'starch/ sucrose metabolic process', 'polysaccharide catabolic process', etc. (Supplemental information 6). These genes were at the highest expression level in FB compared to DF and CM. Cluster 7, with 280 transcripts, had a sudden increase in expression upon transition from $\mathrm{CM}$ to $\mathrm{DF}$, and then remained a steady trend between $\mathrm{DF}$ and $\mathrm{FB}$, consisting of genes mainly involved in 'ribosome', 'ATP catabolic process' and 'protein metabolism process' (Supplemental information 6), such as $\mathrm{Ca}^{2+}$ transporting ATPase (MSTRG.2959) and ATPdependent RNA helicase (MSTRG.10766).

\subsection{Alternative Splicing Prediction}

AS events can increase the complexity and diversity of genes (Wang Y 2015). Some basic AS events, including alternative 3' splice receptor (A3SS), alternative 5' splice donor (A5SS), intron retention (IR) and exon skipping (SKIP) (Wang Y 2015), were investigated in this study. Our data identified 2581 AS events in CM, 2635 in DF and 2203 in FB (Fig. 4A). IR is the major class which accounted for over $44.35 \%$ of these AS events in O. sinensis. When comparing AS events between different growth stages of $O$. sinensis, we found that 946, 652 and 937 DASs related to 783, 566 and 780 DSGs were found to be significantly different in DF vs. CM, in FB vs. DF and in FB vs. CM, respectively (Fig. $4 \mathrm{~B}$ and C, Supplemental information 7). IRrelated DSGs is the major class in all types of DSGs (Fig. 4C, Supplemental information 7). Then the function enrichment analysis of DSGs showed that in DF vs. CM, DSGs were mainly enriched in 'tryptophan/ tyrosine/ L-phenylalanine biosynthetic process'. In FB vs. DF, DSGs were enriched in 'leucine and valine biosynthetic process' (Supplemental information 8). In FB vs. CM, DSGs were enriched in 'damaged DNA binding', 'oxidoreductase activity/ reduction of molecular oxygen' and ' heme binding'. (Supplemental information 8). Moreover, there are 173 shared DSGs among the three growth stages (Supplemental information 9), such as a gene encoding Hsp 90 binding chaperon (mod-E, MSTRG.8149) and a eukaryotic translation initiation factor gene (MSTRG.6510), both of which were significantly up-regulated in FB by 5.24-fold and 5.04-fold compared to CM, respectively.

\subsection{Analysis of cell cytoskeleton genes}

The transcriptome analysis showed that the expression levels of cell cytoskeleton genes were altered during the three serial growth stages. One actin gene (MSTRG.3317, Supplemental information 1) was down-regulated by 3.43-fold in FB compared to DF, and two tubulin / FtsZ protein genes (MSTRG.3823 and MSTRG.6894, Supplemental information 1) were up-regulated by 3.04- and 2.90- fold in FB compared to $\mathrm{DF}$, respectively. TubA and $t u b B$ are essential for mitosis, nuclear migration and ascospore formation (Kirk KE 1991). One actin related protein gene (MSTRG. 3090, Supplemental information 1) was upregulated by 3.66- fold in FB compared to DF, while in DF vs. CM, the gene was down-regulated by about 2.86- fold in DF compared to CM.

Peer] reviewing PDF | (2019:08:40454:3:0:NEW 26 Nov 2019) 
263

264

265

266

267

268

269

270

271

272

273

274

275

276

277

278

279

280

281

282

283

284

285

286

287

288

289

290

291

292

293

294

295

296

297

298

299

300

\subsection{Analysis of development-related genes regulated by environmental factors}

Fruiting body development is a complex cellular differentiation process, which is regulated by special environmental stimulus. In our study, several putatively development-related genes putatively regulated by light were also identified (Supplemental information 10). For examples, one nucleoside diphosphate kinase gene (ndk, MSTRG. 2107), was up-regulated by 6.23-fold in FB compared to DF. Besides, three of superoxide dismutase genes (sod, MSTRG. 2218, MSTRG. 5954 and MSTRG.6951, Supplemental information 10) were remarkably up-regulated in FB compared to DF. Our data showed that the expression level of COX gene (MSTRG.3789, Supplemental information 10) increased in FB at 6.44- fold higher expression level than that in DF. Besides, our data showed that the expression level of the NADPH oxidase gene (MSTRG.7071, Supplemental information 10) was significantly higher in FB at 4.06-fold of DF and 4.08-fold of CM.

In addition, low temperature is another physical factor influencing fruiting body development. Several genes regulated by cold were identified in this study, such as $\beta$ subunit of fatty acid synthase gene (cel-2, MSTRG.6193, Supplemental information 10) and acetyl-CoA desaturase gene (MSTRG.3201, Supplemental information 10). In our study, the expression level of cel-2 and acyl-CoA desaturase gene were up-regulated in FB by 6.36-fold and 6.42-fold compared to CM, respectively. In FB vs. DF, acyl-CoA desaturase gene was expressed at 6.52-fold higher level in FB than that in DF (Supplemental information 10). Besides, two genes (MSTRG.4136, MSTRG.10796, Supplemental information 10) encoding lowtemperature enzyme were potentially identified to be heat-shock protein (Hsp) 70 chaperone, which was supported by the evidence that, Mod-E, a Hsp 90 homolog, was reported to be involved in the temperaturedependent sexual development in P. anserina (Loubradou G 1997). Moreover, four heat shock protein 101 genes (MSTRG.11220, MSTRG.10780, MSTRG.10779 and MSTRG.1122, Supplemental information 10) were found to be significantly up-regulated in FB and DF compared to CM.

\subsection{Analysis of signal transduction pathway}

The fruiting body development was regulated by specific environmental stimuli, and subsequently regulated by signal transduction pathways. In our study, a total of 13 genes encoding MAPKKK, MAPKK and MAPK were identified in the transcriptome during the growth periods. Our data found that four of five MAPKKK encoding genes (MSTRG.10919, MSTRG.5562, MSTRG. 8422 and MSTRG.3582, Supplemental information 1), were expressed at higher levels at stages of DF and FB compared to CM. MSTRG.6508 was annotated as MAPK, and MSTRG.3890 as WD domain containing protein, a MAPK binding protein. Both MSTRG.3890 and MSTRG. 6508 were expressed at higher levels in FB compared to CM (Supplemental information 10). Some serine / threonine-protein kinase genes (e.g. MSTRG.9127, MSTRG.6087, MSTRG.3872 and MSTRG.3872, FDR > 0.001, Supplemental information 1) were at the tendency of higher expression levels in FB and DF compared to CM. Afterwards, these activated MAPK protein would activate the downstream transcript Factors (TFs) to regulate the fruiting body development. Additionally, PKA pathway also leads to ascocarp formation and is associated with the asexual development, hyphal growth, and pathogenicity in several Ascomycetes species (Pöggeler 2006). One cAMP-dependent protein kinase encoding gene (MSTRG.2256, Supplemental information 1) is up-

Peer] reviewing PDF | (2019:08:40454:3:0:NEW 26 Nov 2019) 
301

302

303

304

305

306

307

308

309

310

311

312

313

314

315

316

317

318

319

320

321

322

323

324

325

326

327

328

329

330

331

332

333

334

335

336

337

338

339

regulated by 2.34-fold in CM compared to DF. Taken together the fruiting body development in O. sinensis might be more dependent on the MAPK pathway than the cAMP - dependent PKA pathway.

\subsection{Validation of Transcriptome data by quantitative RT-PCR analysis}

To confirm the reliability of RNA-Seq analysis, the expression level of the genes were randomly selected and analyzed by qPCR during three development stages. In FB vs. DF, except for two genes (MSTRG.5396 and MSTRG.5638), the expression changes of eight genes detected by qPCR is similar to the direction of fold change acquired by the Illumina sequencing results, although the fold changes detected by qPCR wasn't completely consistent with those determined by RNAseq analysis, including six genes upregulated in FB and two genes up - regulated in DF (Fig. 5A). In DF vs. CM comparison, except for two genes (MSTRG.5638 and MSTRG.5297, Supplemental information 1), the qPCR results of seven genes were similar to the results of transcriptome analysis, including four genes up-regulated in $\mathrm{CM}$, two gene upregulated in DF and one genes similarly expressed between the two stages (Fig. 5B). These results showed that our transcriptome data are reliable.

\section{Discussion}

Here, we report the transcriptomes of three different growth stages in O. sinensis. The DEGs analysis revealed that the expression patterns of the fruiting body developmental stages (FB and DF) were more similar compared to the vegetative CM stage. Moreover, the percentage of up-regulated DEGs enriched in ATP process, MCM complex and DNA replication is much higher than that of the down-regulated genes in DF compared to $\mathrm{CM}$, indicating that the increased demand of energy and protein for fruiting body formation. The percentages of DEGs enriched in ribosomal biogenesis and translation were much higher than that of the down-regulated in FB compared to DF. Previous studies indicated that growth media lacking tryptophan or histidine would lead to loss of cleistothecia formation in Aspergillus nidulans (Eckert SE 1999; EckertSE 2000; GH 2001). Moreover, deletion of the tryptophan synthase-encoding gene trpB, or the histidine biosynthesis gene hisB leads to loss of cleistothecia production on medium with low levels of tryptophan or histidine, respectively (EckertSE 2000; GH 2001). These results indicated that the upregulation of ribosome constituent / biogenesis might be prepared for more proteins synthesis and the increased demand of proteins during the stage of fruiting body maturation. In FB vs. CM, we found DEGs were enriched in carbohydrate metabolic process and pyridine - containing / organic hydroxy compound biosynthetic process. Carbohydrates are one important constituent of the fungal cell wall, like $\alpha$-1,3-glucan (ZonneveldBJM 1972). It was proposed that carbohydrates were stored during vegetative growth to be utilized as a carbon source for sexual development (Bart J Janssen 2008; Martin F 2010). Some study showed that higher metabolic demand of fruiting body development in N. crassa (Martin F 2010). Hence, in $O$. sinensis, carbohydrates might be required for mycelium growth, as well as a preparation for the later fruiting process.

PCA analysis showed that the expression profiles at fruiting body development stages (FB and DF) more closely resembled each other than that of CM, consistent with the result of DEGs analysis. Furthermore, hierarchical clustering analyses of all these DEGs across different growth stages showed that genes involved in ribosomal structure/ biogenesis, translation, starch/ sucrose metabolic process and

Peer) reviewing PDF | (2019:08:40454:3:0:NEW 26 Nov 2019) 
340

341

342

343

344

345

346

347

348

349

350

351

352

353

354

355

356

357

358

359

360

361

362

363

364

365

366

367

368

369

370

371

372

373

374

375

376

377

378

379

polysaccharide catabolic process were at the highest expression level in FB compared to DF and CM, indicating that more new protein synthesis might be needed for fruiting body maturation. On the other hand, starch and sucrose metabolism are associated with the production of energy and active compounds, such as sugars and volatile secondary metabolites in fruiting body (Bart J Janssen 2008). Six genes involved in 'starch metabolic process' were at significant and the highest expression level in FB, including glycogen synthase gene (MSTRG.2946), a putative glycoside hydrolase gene (MSTRG.3495), and one gene encoding glucoamylase I precursor (MSTRG.5107), etc. Polysaccharide, an active metabolite, has been reported to be important growth factors in fungi, like Tuber melanosporum (Tisserant E 2011) and Trichoderma reesei (Martinez D 2008). Polysaccharides from the fruiting body of Cordyceps militaris have been proven to have antioxidant - and immune - stimulating activities (Yu R 2007; Lee JS 2011). These results revealed more energy consumption and secondary metabolites production in FB compared to DF and CM. Besides, genes involved in ATP catabolic process were found to be at much higher expression level in the stages of fruiting body formation (DF and FB) compared to CM. Previous studies showed that ATP-dependent helicase mutant of Cordyceps militaris showed poor production of fruiting body (Zheng Z L 2015). And $\mathrm{Ca}^{2+}$ signaling pathway ( $\mathrm{Ca}^{2+}$ transporting ATPase included) was found to be important for fruiting body maturation in Hypsizygus marmoreus (Chen H 2018). Mutants in several subunits of complex 1 of respiration chain composed of proton-pumping NADH ubiquinone oxidoreductase were sterile (Fecke W 1994), which displayed similar phenotype with respect to the lack of energy. Hence, these results revealed an increased demand of energy and metabolites in fruiting body development.

Alternative splicing contributes to protein diversity. In this study, some basic AS events, including exon skipping (SKIP), intron retention (IR), alternative 3' splice receptor (A3SS) and alternative 5' splice donor (A5SS) (Wang Y 2015), were analyzed. IR is the major event in O. sinensis, similar to that in Cordyceps militaris (Yalin Yin 2012), while lower than that in Aspergillus oryzae (91.56\%)(Wang B 2010) and Tuber melanosporum (94\%) (Tisserant E 2011). Moreover, IR-related differential splicing genes (DSGs) are the major class in all types of DSGs, indicating that IR might play an important role in transcriptional regulation during development. Besides, 173 shared DSGs among the comparisons were identified, such as Hsp 90 binding chaperon (mod-E, MSTRG.8149) and a eukaryotic translation initiation factor gene (MSTRG.6510). Mutation of mod-E affects the sexual cycle and suppresses vegetative incompatibility in Podospora anserina (Loubradou G 1997). More recently, CG methylation was found to primarily plays a repressive role on expression in two Pleurotus mushroom species (Jiawei Wen 2019). So differential splicing genes and transcriptional regulation might play significant roles in fruiting body development and require further investigation of transcriptional regulatory mechanisms during development in $O$. sinensis.

Fruiting body formation is accompanied by many morphologically distinct cellular differentiations (Pöggeler 2006). Some genes for cell cytoskeleton structure and organizations have been proven to contribute to sexual differentiation in A. nidulans and P. anserina (Bouhouche K 2004; Upadhyay S 2008). Our data found that the expression levels of some cell cytoskeleton genes were much higher in both FB and $\mathrm{CM}$ than that in DF, such as one actin gene and two tubulin / Fts Z protein gene. Previous studies found that both actin and actin-binding protein fimbrin are critical in the growth of hyphae in A. nidulans (Elena Shestakova 2001; Upadhyay S 2008). Hence cell cytoskeleton genes might play an important role in vegetative growth and sexual development in O. sinensis.

Peer] reviewing PDF | (2019:08:40454:3:0:NEW 26 Nov 2019) 
O. sinensis exclusively distributes in the harsh alpine environment of the Tibetan Plateau of Asia. Fruiting body development is also regulated by the special ecological stimulus. In general, the mycelia of O. sinensis grows in the dark, while fruiting body grows under the light to maturity (Xin Zhong 2018), indicating that light is required for fruiting body maturation. Previous studies showed that blue light initiates fruiting body formation in Coprinopsis cinerea and Schizophyllum commune (R 1985; Purschwitz J 2006). The molecular mechanism underlying light-regulated fruiting body development has been partly elucidated in some basidiomycetes, such as Aspergillus nidulans (Bayram O"2010) and Neurospora crassa (Oda K 1997). In this study, several light- regulated genes were also identified, such as nucleotide diphosphate kinase gene $(n d k), \mathrm{NADPH}$ oxidase gene (nox), superoxide dismutase gene (sod) and cytochrome oxidase gene (cox). Mutants of the NADPH oxidase gene, noxA, were found to be sterile in Aspergillus nidulans (LaraOrtíz T 2003). In P. anserina, the mutant of PaNox1, the ortholog of noxA, no longer developed to mature fruiting bodies (Malagnac F 2004). Both paNox1 and paNox2 were also required for controlled production of superoxide and peroxide during sexual development (Malagnac F 2004). These results demonstrated that Nox catalyzed production of ROS, which is critical for sexual fruiting body development in filamentous fungi. Besides, SOD-1 was required for correct fruiting body morphology in N. crassa and it could be due to that SOD-1 is involved in generating a light-dependent ROS gradient that controls neck positioning(Yoshida Y 2004). Besides, mutant of cytochrome oxidase (COX) gene showed the delayed perithecial formation and reduced ascus production in Podospora anserina, indicating that it is related to sexual development (Stumpferl SW 2004). Both COX and SOD regulate the level of ROS, suggesting that ROS gradient might participate into fruiting body development. NDK was required for light-dependent neck positioning on the perithecia in N. crassa and possibly involved in a light signal transduction pathway (Yasunobu Ogura 2001). To summarize, light impacts the fruiting body development in O. sinensis, which might be mediated by ROS pathway. Further study is required to investigate the molecular mechanisms underlying ROS - mediated fruiting body development in O. sinensis. Besides, some cold - regulated genes were identified, such as fatty acid synthase gene ( cel - 2), acyl - CoA desaturase gene, two potentially encoding heat-shock protein (Hsp) 70 chaperone genes, and four Hsp 101 genes. A previous study showed that cel-2 mutant of $N$. crassa led to fewer perithecia or rare ascospore (Marta Goodrichtanrikulu 1999). Mod-E, a Hsp 90 homolog, was reported to be involved in the temperature-dependent sexual development in P. anserina (Loubradou G 1997).However, few related studies were reported (Pöggeler 2006). Therefore, it remains to be determined whether mod-E or other (heat-shock) proteins are involved in temperaturedependence of fruiting body development and the molecular mechanism.

The fruiting body development was a complex cellular differentiation process, which was regulated by specific environmental stimuli, and subsequently regulated by signal transduction pathways. Mitogen-activated protein kinase (MAPK) modules have been identified in N. crassa by genome analysis (Borkovich KA 2004). MAPK module includes three kinases that establish a sequential activation pathway comprising a MAPK kinase kinase (MAPKKK), MAPK kinase (MAPKK), and MAPK (Kronstad J 1998; Widmann C 1999), among which three different MAPKs and two different MAPKKKs have been shown to be involved in the fruiting body development in different mycelial ascomycetes (Pöggeler 2006). Some MAPKKK cascade protein kinase, like $A$. nidulans SteC, regulate conidiophore development and are required for cleistothecial development 
420

421

422

423

424

425

426

427

428

429

430

431

432

433

434

435

436

437

438

439

440

441

442

443

444

445

446

447

448

449

450

451

452

453

454

455

456

(Wei H 2003). In plant pathogenic fungus, Magnaporthe grisea and Fusarium graminearum, the homologs of MAPKKK protein genes, are essential for plant infection and female fertility (Hou Z 2002). In our data four of five MAPKKK genes were up regulated in the stages of fruiting body formation (FB and $\mathrm{DF}$ ) compared to $\mathrm{CM}$. Two WD domain containing protein (MAPK binding protein) genes were expressed at much higher levels in FB than that in CM. Cpc 2 encoding WD protein has been proven to control sexual development and amino acid biosynthesis in N. crassa (Müller F 1995). Additionally, genes encoding serine / threonine-protein kinase, which act upstream of MAK-2 during fruiting body development through regulating conidiophore development, are essential for cleistothecial development (Pandey A 2004). Besides our data found that one cAMPdependent protein kinase gene was up-regulated in CM compared to DF, suggesting that the PKA signal pathway might play roles in the vegetative growth of $O$. sinensis. However, it needs more data to prove it. To summarize, the fruiting body development in $O$. sinensis might be more dependent on MAPK cascade signaling pathway than on PKA pathway, which is similar to C. militaris (Yalin Yin 2012). Further studies are needed to investigate the MAPK-dependent pathway of fruiting body development in $O$. sinensis.

\section{Conclusion}

In this study, Illumina sequencing was performed to investigate the transcriptomes from three serial growth stages of artificially-cultivated $O$. sinensis (CM, DF and FB), which provided large numbers of transcripts to investigate the biology of fruiting body development. The transcriptomic profiles of the stages of fruiting body development (FB and DF) closely resembled compared to the undifferentiated CM stage. Hierarchical clustering analysis and GO enrichment analysis indicated that fruiting body formation is energy and protein intensive. And more various proteins, secondary metabolites and active compound might be produced in mature fruiting body. Furthermore, some ecological factors regulated genes were identified, suggesting that light-regulated fruiting body development might be via ROS-mediated pathway. For the first time, the altered expression levels of some cytoskeleton genes across the three growth stages were identified in this study. Besides, differentially splicing genes might play an important role in fruiting body development of $O$. sinensis. Overall, our work provided a comprehensive overview into fruiting body development of $O$. sinensis and built a foundation for unraveling molecular basis of fruiting body development, which will help the large-scale cultivation of $O$. sinensis in the future.

Abbreviations: FB: mature fruiting body of $O$. sinensis, CM: the mycelium of $O$. sinensis; DF: developing fruiting body of $O$. sinensis ; NGS: next-generation sequencing; RNAseq: RNA-sequencing; $\log _{2} \mathrm{FC}: \log _{2}$ foldchange; DEG: differentially expressed genes; SEG: specifically expressed genes; EC: enzyme codes. PDA: potato dextrose agar; SRR: clean reads of O. sinensis from the Sequence Read Archive; AS: Alternative splicing; SKIP: exon skipping; IR: retention of single/multiple intron; A3SS / A5SS: alternative 3' splice receptor/ alternative 5' splice donor; DSG: differential splicing genes; DAS: differential alternative splicing.

Peer) reviewing PDF | (2019:08:40454:3:0:NEW 26 Nov 2019) 
457

458

459

460

461

462

463

464

465

466

467

468

469

470

471

472

473

474

475

476

477

478

479

480

481

482

483

484

485

486

487

488

489

490

491

492

493

494

495

496

Authors' contributions: Jinlin Guo conceived this study, designed the experimental plan. Cheng Peng participated in designing the experiment. XinxinTong performed data analysis, writing-original draft. Han Zhang participated in data analysis and performed qPCR experiment. Zhengyao Xue revised and reviewed the manuscript. Jing Cao and Fang Wang participated in sample preparing, sample processing and total RNA extracting. All authors read and approved the final manuscript.

Acknowledgments: This study was supported by the Natural Sciences Foundation of China Science (81872959, 81373920, 30801522). Yong Science and Technology Innovation Team of Sichuan Province (2019CXTD0055), China Scholarship Foundation (201708510027)

Competing interests: The authors declare that they have no competing interests.

Supplementary Materials: Supplemental information 1 Sequence annotations and gene expression profiling, Supplemental information 2 The shared DEGs between these growth stages, Supplemental information 3 GO enrichment of significantly differentiated expressed genes, Supplemental information 4 Differentially expressed genes involved in enriched GO terms between stages, Supplemental information 5 Heatmap analysis of DEGs between different growth stages, Supplemental information 6 GO enrichment of the DEGs in different clusters, Supplemental information 7 Statistics of AS events in different growth stages, the number of DASs and DSGs between different growth stages of O. sinensis, Supplemental information $8 \mathrm{GO}$ enrichment of different splicing genes between different growth stages, Supplemental information 9 All shared AS genes among all different comparisons of these growth stages, Supplemental information 10 Fruiting-body development candidate genes, Supplemental information 11 Primers for quantitative RT-PCR.

\section{References}

Ashburner M, B. C., Blake JA, Michael Ashburner, Catherine A Ball, Judith A Blake, David Botstein, Heather Z Butler, J Michael Cherry, Allan Peter Davis, Kara Dolinski, Selina S Dwight, Janan T Eppig, Midori A Harris, David P Hill, Laurie Isseltarver, Andrew Kasarskis, Suzanna E Lewis, John C Matese, Joel E Richardson, Martin Ringwald, Gerald M Rubin, Gavin Sherlock (2000). "Gene ontology: tool for the unification of biology. The Gene Ontology Consortium. " Nat Genet 25: 25-29.

Bart J Janssen, K. T., Robert J Schaffer, Rob Alba, Lena Balakrishnan, Rebecca Kent Bishop, Judith H Bowen, Ross N Crowhurst, Andrew P Gleave, Susan E Ledger, Steve Mcartney, Franz B Pichler, Kimberley C Snowden, Shayna Ward. (2008). " Global gene expression analysis of apple fruit development from the floral bud to ripe fruit." BMC Plant Biology 8(1): 16-16.

Bayram O” , B. G., Fischer R, Rodriguez-Romero J. (2010). " Spotlight on Aspergillus nidulans photosensory systems." Fungal Genet Biol 47: 900-908.

Cole Trapnell, Brian A Williams, Geo Pertea, Ali Mortazavi, Gordon Kwan, Marijke J Van Baren, Steven L Salzberg, Barbara J Wold, Lior Pachter. (2010). "Transcript assembly and quantification by RNA-Seq reveals unannotated transcripts and isoform switching during cell differentiation." Nature Biotechnology (5): 511-515.

Bouhouche K, Z. D., Debuchy R, Arnaise S. (2004). " Altering a gene involved in nuclear distribution increases the repeat-induced point mutation process in the fungus Podospora anserina. ." Genetics 167: 151-159.

Busch S, E. S., Krappmann S, Braus GH. "COP9 signalosome is an essential regulator of development in the filamentous 
497

498

499

500

501

502

503

504

505

506

507

508

509

510

511

512

513

514

515

516

517

518

519

520

521

522

523

524

525

526

527

528

529

530

531

532

533

534

535

536

537

fungus Aspergillus nidulans." Mol Microbiol 49: 717-713.

Chen Hui, Haibo. Hai, Wang Hong, Wang qiao, Chen Mingjie, Feng Zhiyong, Ye Ming, Zhang jinjing. (2018). " Hydrogen-rich water mediates redox regulation of the antioxidant system, mycelial regeneration and fruiting body development in Hypsizygus marmoreus." Fungal Biology 122(5): 310.

Ding B, L. M. A., Wang H, Cheng-Hua Zhao, ChristerLöfstedt (2011). "Terminal fatty-acyl-CoA desaturase involved in sex pheromone biosynthesis in the winter moth (Operophtera brumata)." Insect Biochemistry and Molecular Biology 41(9): 715-722.

Eckert SE, H. B., Wanke C, Braus GH. (1999). "Sexual development of Aspergillus nidulans in tryptophan auxotrophic strains." Arch Microbiol 172: 157-166.

EckertSE, K., Hoffmann B, Braus GH. (2000). "The tryptophan synthase-encoding trpB gene of Aspergillus nidulans is regulated by the cross-pathway control system " Mol Gen Genet 263: 867-876.

Elena Shestakova, R. H. S., John Condeelis. (2001). "The physiological significance of beta-actin mRNA localization in determining cell polarity and directional motility." Proc Natl Acad Sci USA 98(13): 7045-7050.

Enhua Xia, D. Y., Jianjun Jiang, Qunjie Zhang, Yuan Liu, Yunlong Liu, Yun Zhang, Haibin Zhang, Cong Shi, Yan Tong, Changhoon Kim, Hua Chen, Yanqiong Peng, Yue Yu, Wei Zhang, Evan E Eichler, Lizhi Gao. (2017). "The caterpillar fungus, Ophiocordyceps sinensis, genome provides insights into highland adaptation of fungal pathogenicity." Scientific Reports 7(1): 1806-1817.

Fecke W, S. V., Ohnishi T, Weiss H. (1994). " Disruption of the gene encoding the NADH-binding subunit of NADH: ubiquinone oxidoreductase in Neurospora crassa. Formation of a partially assembled enzyme without FMN and the iron-sulphur cluster N-3." Eur J Biochem 220: 551-558.

GH, B. (2001). "Regulation of the Aspergillus nidulans hisB gene by histidine starvation." Curr Genet 38: $314-322$.

Hou Z, X. C., Peng Y, Katan T, Kistler HC, Xu JR. (2002). "A mitogen-activated protein kinase gene (MGV1) in Fusarium graminearumis required for female fertility, heterokaryon formation, and plant Mol Plant" Microbe Interact 15: 1119-1127.

Jiawei Wen, Z. Z., Lei Gong, Hongwei Xun, Juzuo Li, Bao Qi, Qi Wang, Xiaomeng Li, Yu Li, Bao Liu (2019). "Transcriptome Changes during Major Developmental Transitions Accompanied with Little Alteration of DNA Methylome in Two Pleurotus Species." Genes 10(6): 465-479.

Janssen B J, T. K., Schaffer R J. (2008). " Global gene expression analysis of apple fruit development from the floral bud to ripe fruit." BMC Plant Biology 8(1): 16-.

Jin Xu, Y. H., Xiangxiang Chen, Minghe Mo. (2016). " The Mechanisms of Pharmacological Activities of Ophiocordyceps sinensis Fungi." Phytotherapy Research 30(10): 1572-1583.

Jinlin Guo, X. y. L., Kahoru Kanari. (2012). "Towards sustainable livelihoods from wild medicinal resources: Economic aspects of harvesting and trading the Chinese Caterpillar Fungus Ophiocordyceps sinensis and Southern Schisandra sphenanthera in China's Upper Yangtze Ecoregion" TRAFFIC Bulletin 42(1).

Kanehisa M, G. S. (2000). "KEGG: kyoto encyclopedia of genes and genomes." Nucleic Acids Res 28: 27-30.

Kim, D., B. Langmead, S.L. Salzberg. (2015). "HISAT: a fast spliced aligner with low memory requirements." Nature methods 12(4): 357-360.

Kirk KE, M. N. (1991). "The tubB alpha-tubulin gene is essential for sexual development in Aspergillus nidulans." Genes Dev 5: 2014-2023.

Kronstad J, d. M. A., Funnell D, Laidlaw RD, Lee N, de Sa MM, Ramesh M. (1998). "Signaling via cAMP in fungi: interconnections with mitogen-activated protein kinase pathways." Arch Microbiol 170: 395-404.

Peer] reviewing PDF | (2019:08:40454:3:0:NEW 26 Nov 2019) 
538

539

540

541

542

543

544

545

546

547

548

549

550

551

552

553

554

555

556

557

558

559

560

561

562

563

564

565

566

567

568

569

570

571

572

573

574

575

576

577

578

Lara-Ortíz T, R.-R. H., Aguirre J. (2003). " Reactive oxygen species generated by microbial NADPH oxidase NoxA regulate sexual development in Aspergillus nidulans." Mol Microbiol 50: 1241-1255.

Lee JS, H. E. (2011). "Immunostimulating activity of the polysaccharides isolated from Cordyceps militaris." Int Immunopharmacol. 11: 1226-1233.

Li B, D. C. N. (2011). " RSEM: accurate transcript quantification from RNA-Seq data with or without a reference genome." Bioinformatics BMC 12(1): 323-323.

Li Xiang, Y. L., Yingjie Zhu, Hongmei Luo, Chunfang Li, Xiaolan Xu, Chao Sun, Jingyuan Song, Linchun Shi, Liu He, Wei Sun, Shilin Chen. (2014). "Transcriptome analysis of the Ophiocordyceps sinensis fruiting body reveals putative genes involved in fruiting body development and cordycepin biosynthesis." Genomic 103(1): 154159.

Lo, H., Hsieh, C., Lin, FY., Hsu TH. (2015). "A systematic review of the mysterious caterpillar fungus Ophiocordyceps sinensis in Dong-Chong Xia Cao (Dong Chong Xia Căo) and related bioactive ingredients." Journal of Traditional and Complementary Medicine 3(1): 16-32.

Loubradou G, B. J., Turcq B. (1997). " A mutation in an HSP90 gene affects the sexual cycle and suppresses vegetative incompatibility in the fungus Podospora anserina." Genetics 147(2): 581-588.

Müller F, K. D., Sattlegger E, Hoffmann B, Ballario P, Kanaan M, Barthelmeß IB. (1995). " The cpc-2 gene of Neurospora crassa encodes a protein entirely composed of WD-repeat segments that is involved in general amino acid control and female fertility." Mol Gen Genet 248: 162-173.

Malagnac F, L. H., Lepere G, Silar P. (2004). " Two NADPH oxidase isoforms are required for sexual reproduction and ascospore germination in the filamentous fungus Podospora anserina." Fungal Genet Biol 41: 982-997.

Marta Goodrichtanrikulu, D. J. J., Allan E Stafford, Jianntsyh Lin, Thomas A Mckeon. (1999). "Characterization of Neurospora crassa mutants isolated following repeat-induced point mutation of the beta subunit of fatty acid synthase." Curr Genet 36(3): 147-52.

Martin F, Kohler A, Murat C, Balestrini R, Coutinho PM, Jaillon O, Montanini B, Morin E, Noel B, Percudani R, Porcel B, Rubini A, Amicucci A, Amselem J, Anthouard V, Arcioni S, Artiguenave F, Aury JM, Ballario P, Bolchi A, Brenna A, Brun A, Buée M, Cantarel B, Chevalier G, Couloux A, Da Silva C, Denoeud F, Duplessis S, Ghignone S, Hilselberger B, Iotti M, Marçais B, Mello A, Miranda M, Pacioni G, Quesneville H, Riccioni C, Ruotolo R, Splivallo R, Stocchi V, Tisserant E, Viscomi AR, Zambonelli A, Zampieri E, Henrissat B, Lebrun MH, Paolocci F, Bonfante P, Ottonello S, Wincker P. (2010). " Perigord black truffle genome uncovers evolutionary origins and mechanisms of symbiosis. Nature, 2010, ." Nature 464: 1033-1038.

Martinez D, Berka RM, Henrissat B, Saloheimo M, Arvas M, Baker SE, Chapman J, Chertkov O, Coutinho PM, Cullen D, Danchin EG, Grigoriev IV, Harris P, Jackson M, Kubicek CP, Han CS, Ho I, Larrondo LF, de Leon AL, Magnuson JK, Merino S, Misra M, Nelson B, Putnam N, Robbertse B, Salamov AA, Schmoll M, Terry A, Thayer N, Westerholm-Parvinen A, Schoch CL, Yao J, Barabote R, Nelson MA, Detter C, Bruce D, Kuske CR, Xie G, Richardson P, Rokhsar DS, Lucas SM, Rubin EM, Dunn-Coleman N, Ward M, Brettin TS. (2008). "Genome sequencing and analysis of the biomass-degrading fungus Trichoderma reesei (syn. Hypocrea jecorina)." Nat Biotechnol 26: 553-560.

Medicinal, W. S. (2002). "mushrooms as a source of antitumor and immunomodulating polysaccharides." Appl Microbiol Biotechnol 60: 258-274.

Pertea M, Kim D, Pertea GM, Leek JT, Salzberg SL. (2016). "Transcript-level expression analysis of RNA-seq experiments with HISAT, StringTie and Ballgown." Nature protocols 11(9): 1650-1667.

Peer) reviewing PDF | (2019:08:40454:3:0:NEW 26 Nov 2019) 
MihaelaPertea, G. P., Corina Antonescu, Tsung-Cheng, Chang, Joshua T, Mendell, Steven L, Salzberg. (2015). " StringTie enables improved reconstruction of a transcriptome from RNA-seq reads." Nature biotechnology 33(3): 290-295.

Oda K, H. K. (1997). "Genetic analysis of signal transduction through light-induced protein phosphorylation in Neurospora crassa perithecia." Mol Gen Genet 256: 593-601.

Pöggeler, S., M. Nowrousian,U.Kück. (2006). Fruiting-Body Development in Ascomycetes, in Growth, Differentiation and Sexuality. Berlin, Heidelberg, Springer

Purschwitz J, M. 1. S., Kastner C, Fischer R. (2006). " Seeing the rainbow: light sensing in fungi." Curr Opin Microbiol 9: 566-571.

Qilian Qin, G. Z., Huan Zhang, Qian Meng, Jihong Zhang, Hongtuo Wang, Lin Miao, Xuan Li. (2018). "Obstacles and approaches in artificial cultivation of Chinese cordyceps." Mycology 9(1): 7-9.

R, D. (1985). " Blue U.V.-light photoreception in fungi." Rev Physiol 23: 935-943

Stumpferl SW, S. O., Osiewacz HD. (2004). "Impact of a disruption of a pathway delivering copper to mitochondria on Podospora anserina metabolism and life span." Eukaryot Cell 3: 200-211.

Tatusov RL, Fedorova ND, Jackson JD, Jacobs AR, Kiryutin B, Koonin EV, Krylov DM, Mazumder R, Mekhedov SL, Nikolskaya AN, Rao BS, Smirnov S, Sverdlov AV, Vasudevan S, Wolf YI, Yin JJ, Natale DA. (2003). "The COG database: an updated version includes eukaryotes." BMC Bioinformatics 4 (41).

Tisserant E, Da Silva C, Kohler A, Morin E, Wincker P, Martin F. (2011). " Deep RNA sequencing improved the structural annotation of the Tuber melanosporum transcriptome." New Phytol 189: 883-891.

Upadhyay S, S. B. D. (2008). "The role of actin, fimbrin and endocytosis in growth of hyphae in Aspergillus nidulans." Molecular Microbiology 68(3): 690-705.

Wang B, G. G., Wang C, Lin Y, Wang X. (2010). " Survey of the transcriptome of Aspergillus oryzae via massively parallel mRNA sequencing." Nucleic Acids Res 38: 5075-5087.

Walser PJ, V. R., AebiM, Kües U. (2003). Extracellular matrix proteins in mushroom development. Kerala, India, Reseach Signpost.

Wang Y, L. J., Huang BO, Xu YM, Li J, Huang LF, Lin J, Zhang J, Min QH, Yang WM, Wang XZ. (2015). "Mechanism of alternative splicing and its regulation (Review)." Biomedical Reports 3(2): 152-158.

Widmann C, G. S., Jarpe MB, Johnson G. (1999). "Mitogen-activated protein kinase: conservation of a three-kinase module from yeast to human." Physiol. Rev 79: 143-180.

Xin Zhong, L. G., Haizhen Wang, Danhong Lian, Yimei Zheng, Sha Zhou, Wei Zhou, Jinlei Gu, Guren Zhang, XinLiu. (2018). "Profile of Ophiocordyceps sinensis transcriptome and differentially expressed genes in three different mycelia, Sclerotium and fruiting body developmental stages." Fungal Biology 122(10): 943-951.

Xin Zhong, L. G., Shaosong Li, Xutian Kan, Guren Zhang, Xin Liu. (2016). "Transcriptome analysis of Ophiocordyceps sinensis before and after infection of Thitarodes larvae." Fungal Biology 120(6): 819-826.

Yalin Yin, G. Y., Yijie Chen, Shuai Jiang, Man Wang, Yanxia Jin, Xianqing Lan, Yi Liang, Hui Sun. (2012). "GenomeWide Transcriptome and Proteome Analysis on Different Developmental Stages of Cordyceps militaris." PLoS ONE 7(12).

Yasunobu Ogura, Yusuke Yoshida, Naoto Yabe, Hasunuma K. (2001). "A point mutation in nucleoside diphosphate kinase results in a deficient light response for perithecial polarity in Neurospora crassa." J Biol Chem 276(24): 21228-21234.

Yoshida Y, H. K. (2004). " Reactive oxygen species affect photomorphogenesis in Neurospora crassa." J Biol Chem 279: 
6986-6993.

621 Yu R, Yang. W., Song L, Yan C, Zhang Z, Zhao Y. (2007). "Structural characterization and antioxidant activity of a polysaccharide from the fruiting bodies of cultured Cordyceps militaris." Carbohydrate Polymers 70: 430-436.

623 Zheng ZL, Qiu XH, Han RC. (2015). " Identification of the Genes Involved in the Fruiting Body Production and Cordycepin Formation of Cordyceps militaris Fungus." Mycobiology 43(1): 37-42.

625 Zonneveld BJM (1972). " Morphogenesis in Aspergillus nidulans. The significance of $\alpha$-1,3-glucan of the cell wall and $\alpha$-1,3-glucanase for cleistothecium development." Biochim Biophys Acta 273: 174-187.

627 


\section{Figure 1}

Analysis of differentially expressed genes (DEGs) between different growth stages.

A.The number of DEGs is shown on the top of histograms. statistics of DEGs from O. sinensis between different growth stages. B. Venn diagram of DEGs comparing between different growth stages from $\mathrm{O}$. sinensis. $\mathrm{FB}$ represents the mature fruiting body. $\mathrm{CM}$ represents the asexual mycelium. DF represents the developing fruiting body. 
A

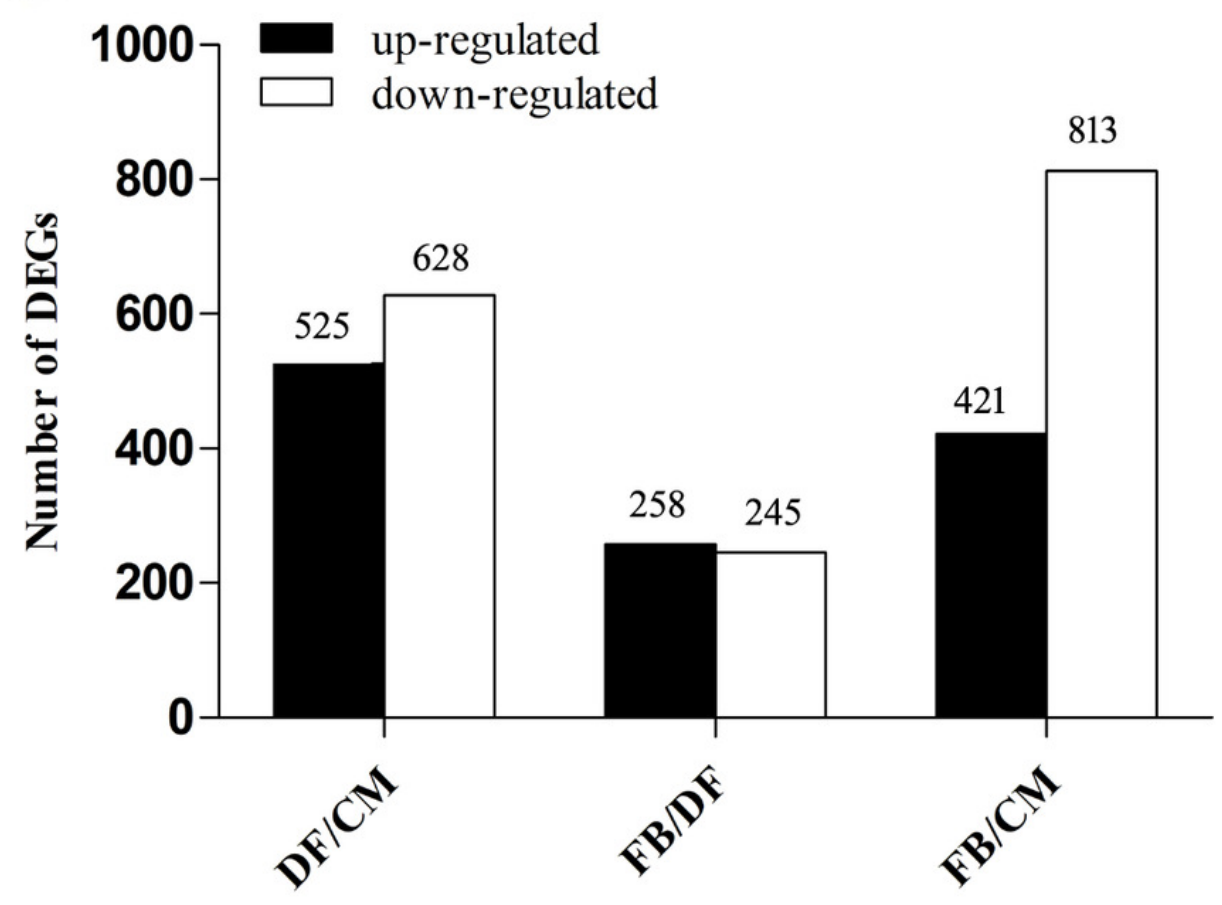

B

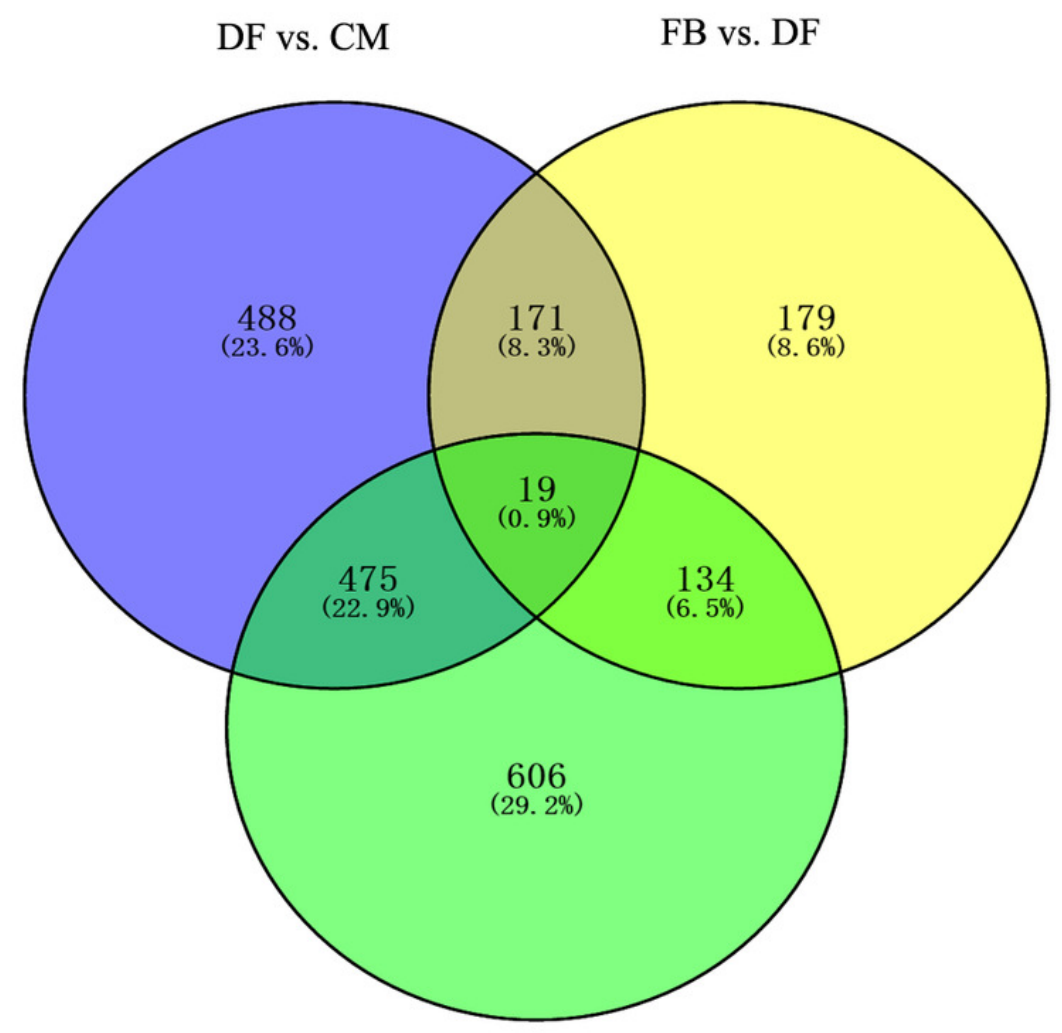

FB vs. CM 


\section{Figure 2}

The most enriched GO functional classification of DEGs between different growth stages.

The most enriched GO functional classification of DEGs between different growth stages. Only the significant GO terms $(p<0.005)$ were shown. X-axes represent the enrichment score (- $\log _{10}$ P-value) of top GO terms enriched among DEGs. The green bars represent cellular components; The orange represent biological processes; the blue bars represent molecular functions. FB represents the mature fruiting body. $\mathrm{CM}$ represents the asexual mycelium. DF represents the developing fruiting body. 
A

\section{DF vs. CM}

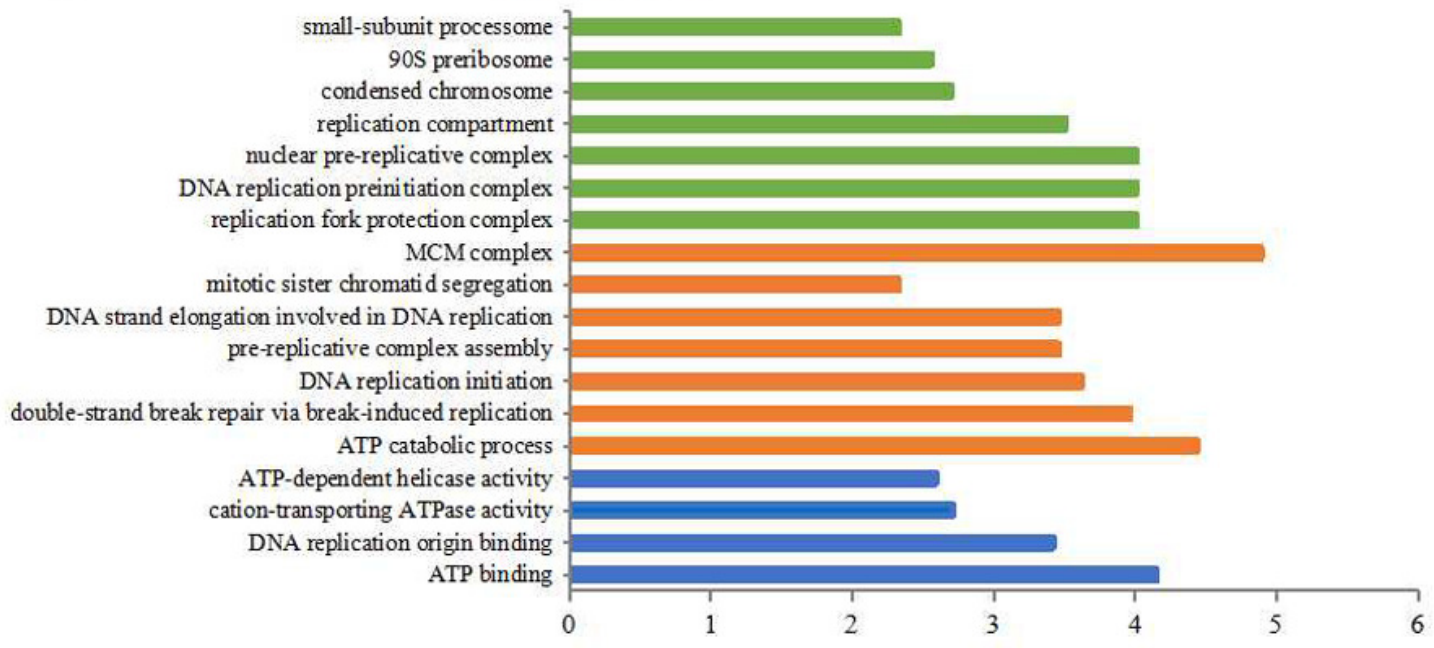

B

FB vs. DF

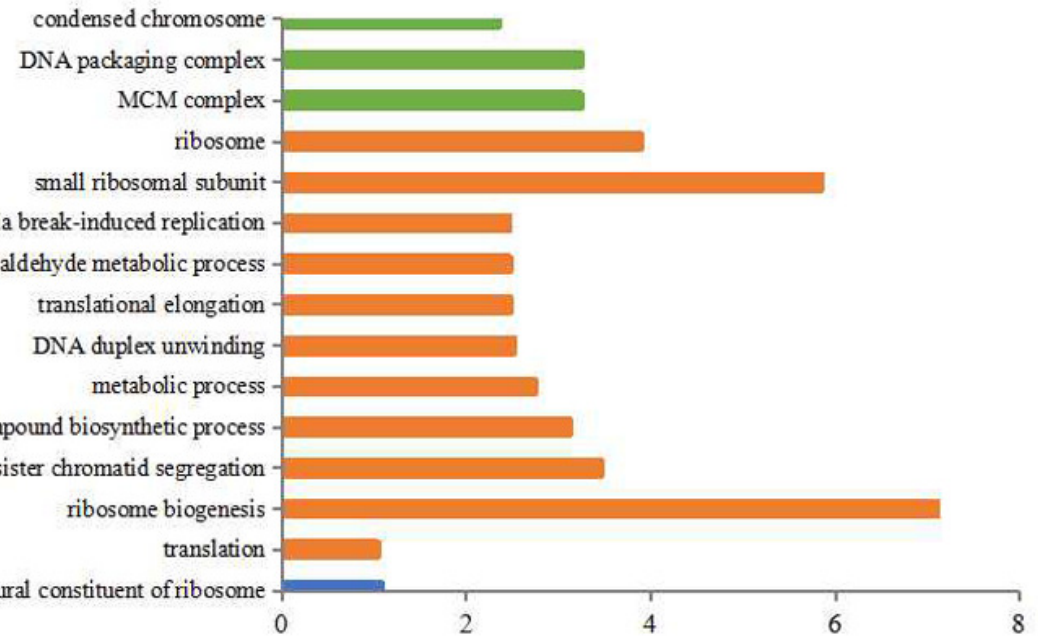

$\mathrm{C}$

\section{FB vs. CM}

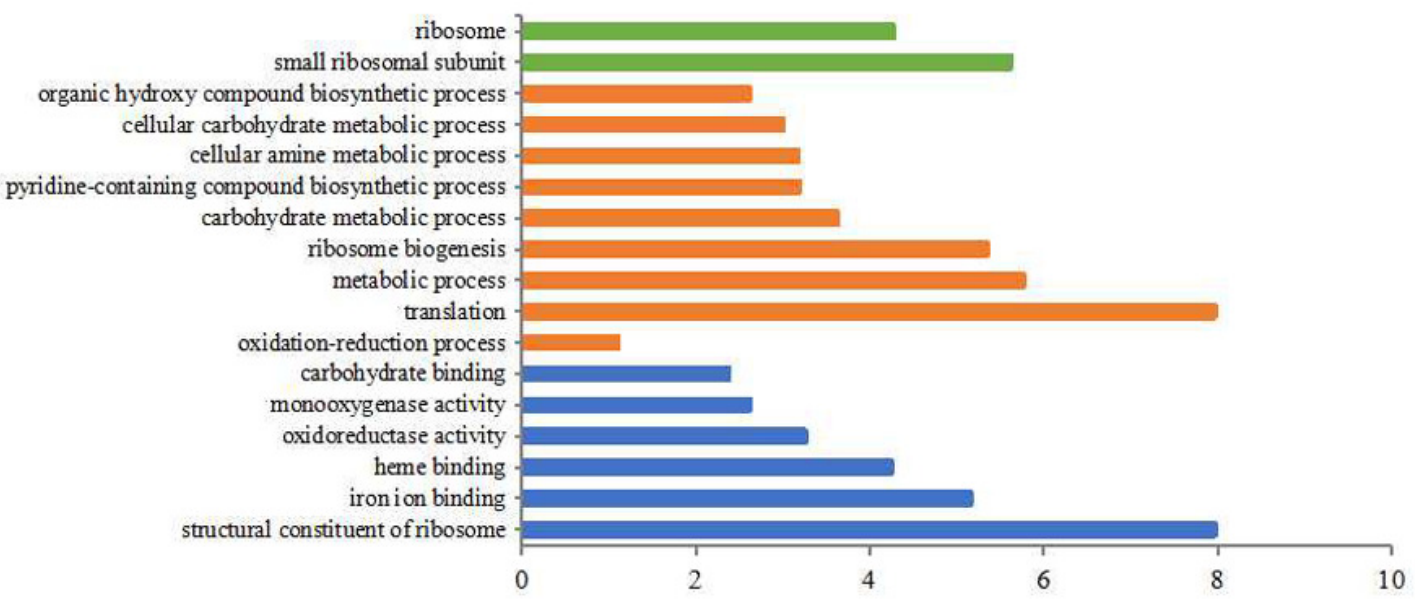




\section{Figure 3}

Clustering of gene expression profiles across the three growth stages.

A. Principle component analysis of the RNA-Seq data. B-H. six gene clusters with different expression patterns. Overlaying curves of all genes within the cluster were shown. FB represents the mature fruiting body. CM represents the asexual mycelium. DF represents the developing fruiting body. 


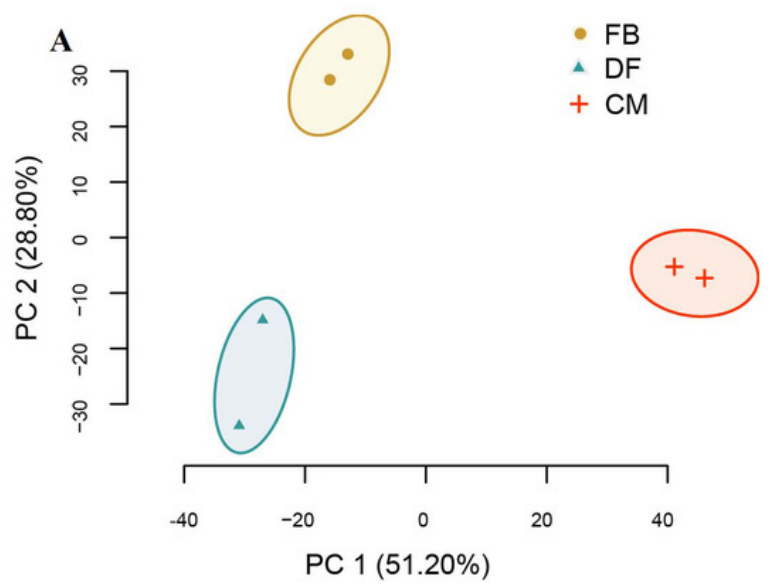

B

C cluster 2, \# 139 genes
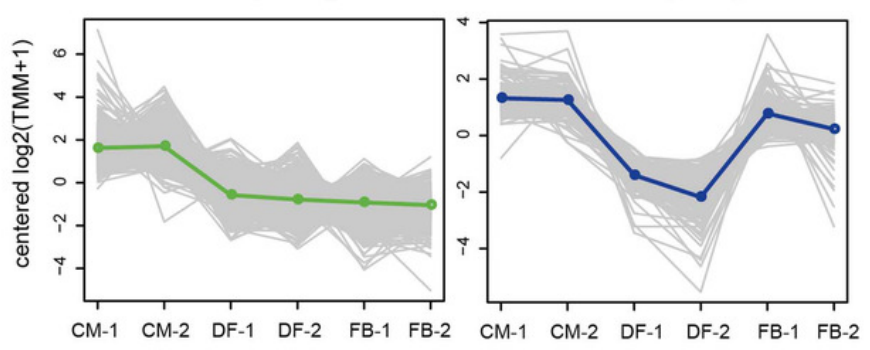

D cluster 3 , \# 72 genes E cluster 4, \# 188 genes
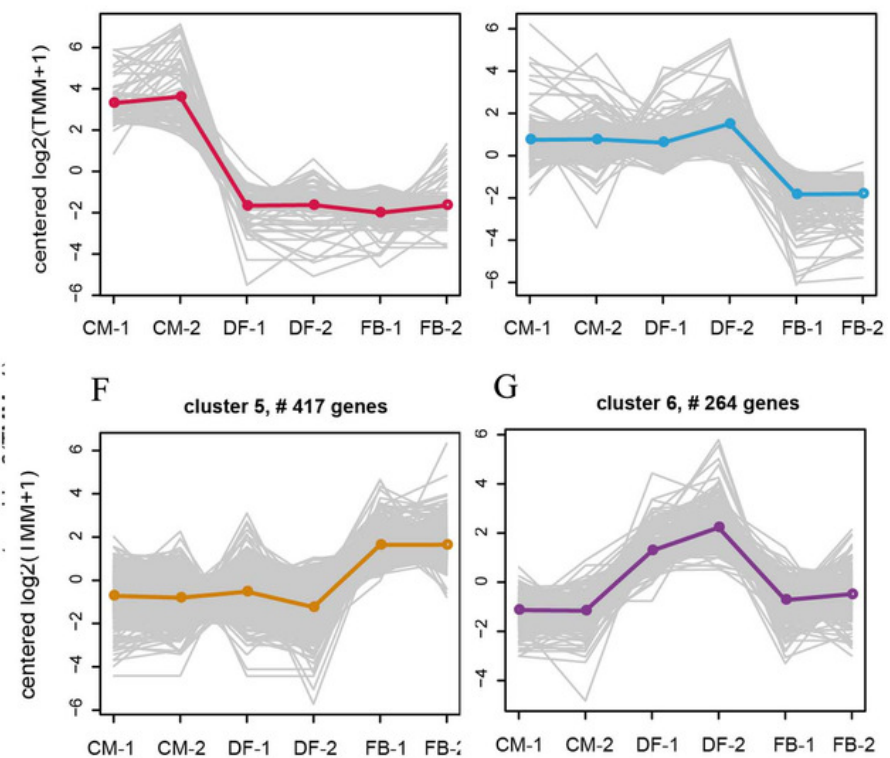

G
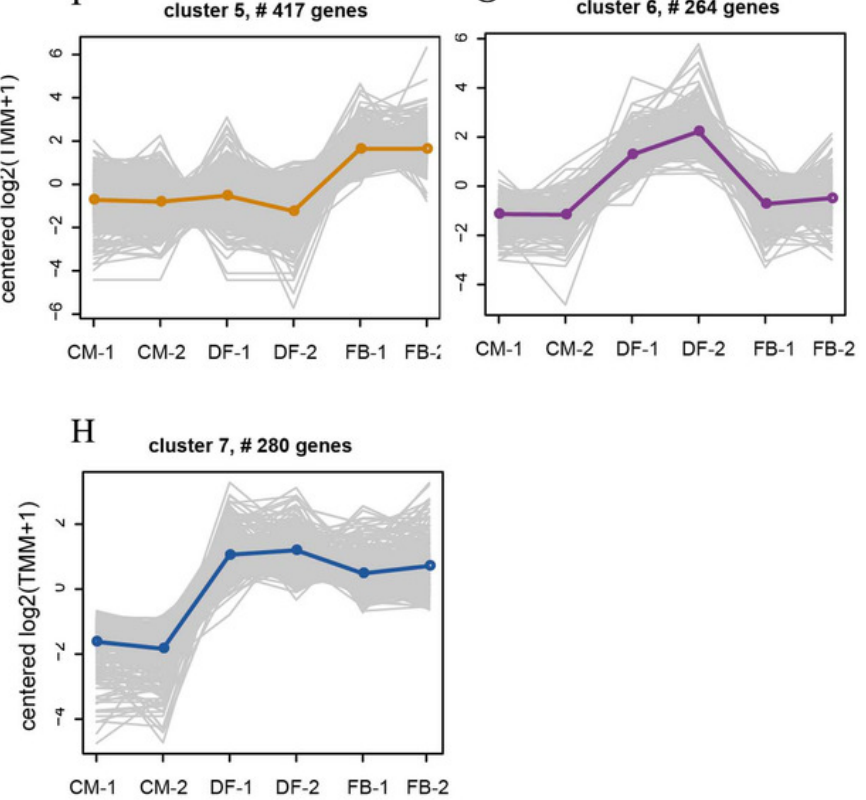

Peer) reviewing PDF | (2019:08:40454:3:0:NEW 26 Nov 2019) 


\section{Figure 4}

AS events in the $O$. sinensis transcriptome.

A. Numbers of alternative splicing (AS) events in different growth stages of $O$. sinensis. B. Numbers of differential alternative splicing events (DASs) between different stages. C. Numbers of differential splicing genes (DSGs) between different stages. The $x$-axis represents AS types. FDR $<0.05$. FB represents the mature fruiting body with mature. CM represents the asexual mycelium. DF represents the developing fruiting body. IR: intron retention, A3SS: alternative 3' splice receptor, A5SS: alternative 5' splice donor, SKIP: exon skipping. 

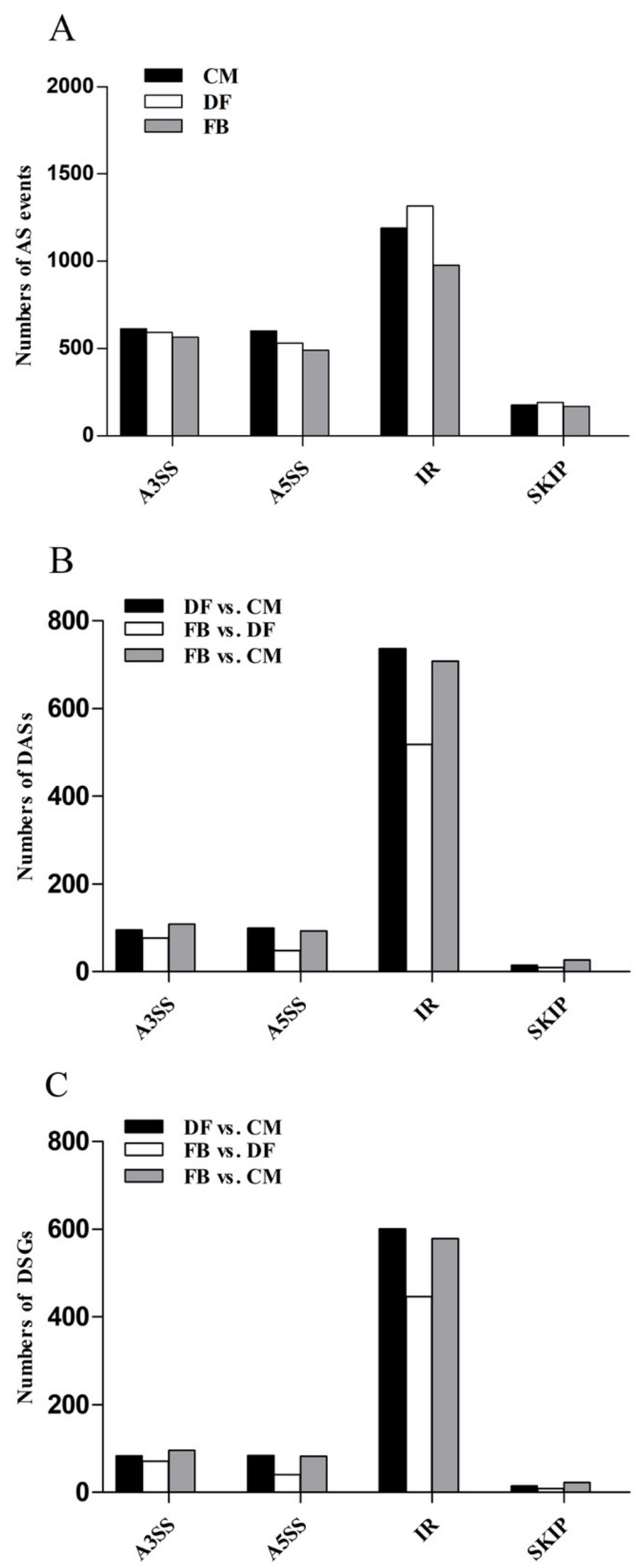

PeerJ reviewing PDF | (2019:08:40454:3:0:NEW 26 Nov 2019) 


\section{Figure 5}

qPCR validation of the expressed genes in Illumina sequencing.

A. Bars represent the fold change in expression of each candidate gene identified in FB relative to DF. B. Bars represent the fold change in expression of each candidate gene identified in CM relative to DF Black bars represent $\mathrm{qRT}$-PCR result $\left(2^{\square \Delta \Delta C t}\right)$. Error bars indicate the standard error. White bars represent the RNA-seq results ( $\log _{2}$ fold change). Histone $\mathrm{H} 2 \mathrm{~A}$ gene ( MSTRG. 2474) was the internal reference. FB represents the mature fruiting body. CM represents the asexual mycelium. DF represents the developing fruiting body. * represents significant difference in gene expression between FB/CM and DF, respectively, with qRT-PCR measured by paired t-test at $p<0.05$ and RNAseq measured by edgeR at FDR $<0.05$.
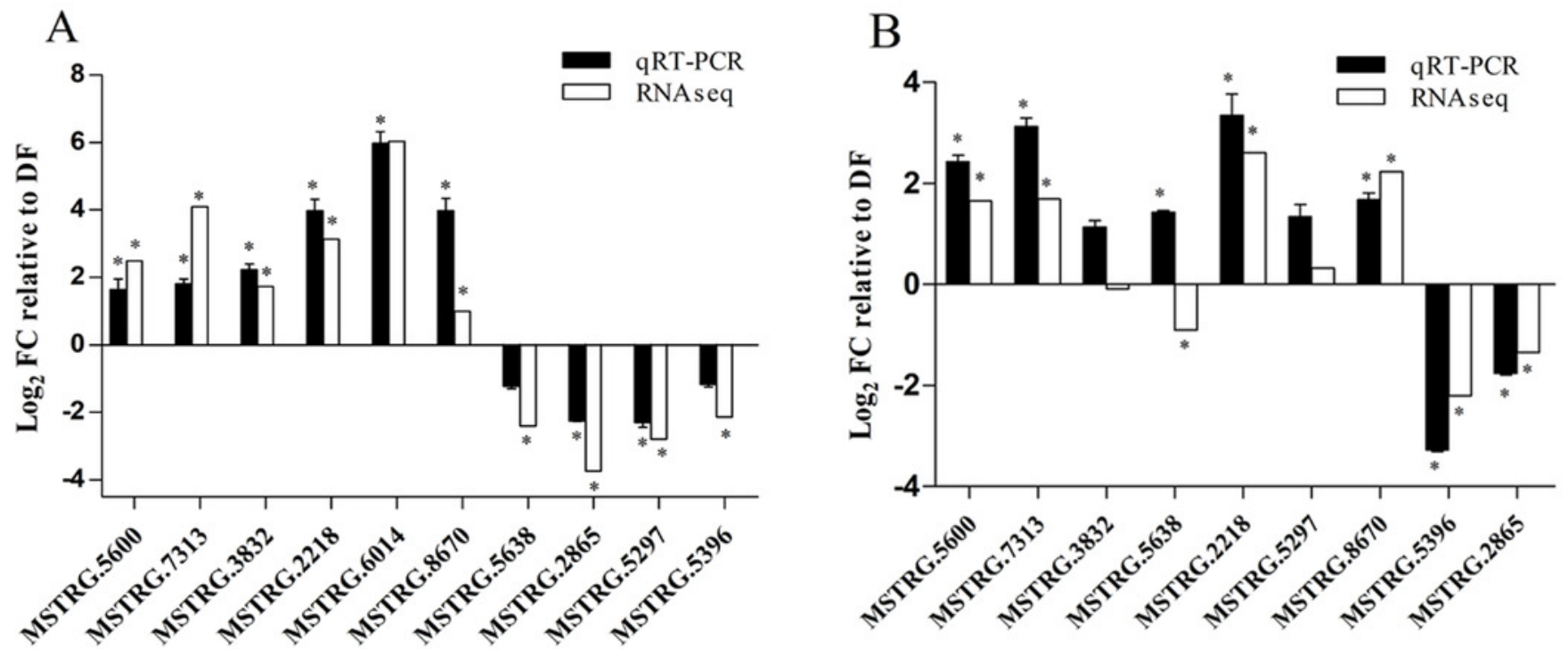


\section{Table $\mathbf{1}$ (on next page)}

Table 1 Mapping results of $O$. sinensis transcriptomes 
1 Table 1 Mapping results of $O$. sinensis transcriptomes.

\begin{tabular}{lllll}
\hline Sample & Clean Reads & Clean bases & GC(\%) & $\begin{array}{c}\text { \% mapped reads } \\
\text { to genome }\end{array}$ \\
\hline CM-1 & $35,128,504$ & $4.43 G$ & 60.40 & 83.41 \\
CM-2 & $36,788,192$ & $4.63 G$ & 60.00 & 83.98 \\
DF-1 & $38,627,930$ & $4.87 G$ & 60.70 & 82.61 \\
DF-2 & $34,270,616$ & $4.32 G$ & 61.00 & 80.77 \\
FB-1 & $32,751,220$ & $4.57 G$ & 60.35 & 85.12 \\
FB-2 & $36,296,836$ & $4.13 G$ & 59.93 & 83.84 \\
\hline
\end{tabular}

